\title{
Review Article \\ Reviewing the Literature on the Effectiveness of Pressure Relieving Movements
}

\author{
Rachel Schofield, Alison Porter-Armstrong, and May Stinson \\ Rehabilitation Sciences and Research Centre, School of Health Sciences, University of Ulster, Jordanstown, BT37 0QB, UK \\ Correspondence should be addressed to Rachel Schofield; schofield-r@email.ulster.ac.uk
}

Received 22 June 2012; Accepted 12 November 2012

Academic Editor: Jeannie Donnelly

Copyright (C) 2013 Rachel Schofield et al. This is an open access article distributed under the Creative Commons Attribution License, which permits unrestricted use, distribution, and reproduction in any medium, provided the original work is properly cited.

Sitting for prolonged periods of time increases seating interface pressures, which is known to increase the risk of developing pressure ulcers. Those at risk of developing pressure ulcers are advised to perform pressure relieving movements such as "pushups" or "forward leans" in order to reduce the duration and magnitude of pressure acting on the vulnerable ischial tuberosity region. The aim of this review was to synthesize and critique the existing literature investigating the effectiveness of pressure relieving movements on seating interface pressures. The twenty-seven articles included in this paper highlight the need for further research investigating the effect of recommended pressure relieving movements on the pressures around the ischial tuberosities. Furthermore, this review found that the majority of individuals at risk of developing pressure ulcers do not adhere with the pressure relieving frequency or magnitude of movements currently recommended, indicating a need for pressure ulcer prevention to be explored further.

\section{Introduction}

Sitting for prolonged periods of time is thought to increase the risk of developing pressure ulcers $[1,2]$. Sitting forces the weight of an individual against the supporting seat surface which compresses the soft tissues around the buttock area between the chair and the bony ischial tuberosities. This pressure causes an obstruction of blood flow that when combined with limited movement, poor sensation, malnutrition, and increased age can eventually lead to ulceration [3-5]. These severe, yet usually preventable wounds are relatively common, spanning acute, rehabilitation, and community settings $[6,7]$, as such, the treatment of pressure ulcers is considered to outweigh the social and financial costs associated with prevention [7].

One of the most effective preventative methods in terms of cost and pressure relief is regular repositioning [8]. Within rehabilitation, individuals at risk of developing pressure ulcers are taught and encouraged to perform regular repositioning movements in order to redistribute the build-up of pressure around the ischial tuberosity and sacral regions. These repositioning movements include vertical pushups, lateral and forward leans. Occupational therapists being responsible for seating and postural care are ideally placed to educate the individual and their carers on good skin health and the importance of relieving pressure at the seating interface regularly [9].

Currently, individuals "at risk" are advised to change their posture by performing pressure relieving movements as often as every 15 to 30 minutes $[10,11]$. However, the evidence that these pressure relieving movements effectively redistribute pressures between the individual and the seating surface, known as seating interface, has not been explored. The aim of this review was to synthesize and critique the existing literature on the effectiveness of pressure relieving activities on seating interface pressures.

\section{Materials and Methods}

An electronic search using a range of databases (AMED, CINAHL, Cochrane, Embase, and Medline) was conducted for the years 2002-2012. A combination of keywords was used including seating; sitting; interface pressure; pressure ulcer; decubitus ulcer; activities of daily living; movement; pressure relief; posture; weight shift; reposition; tilt and recline. 
Articles were selected if they were written in English and described experimental research in which functional body movement (both passive and active) was manipulated and seating interface pressures were measured. Studies which did not investigate pressure relieving movements or activities were excluded. No limits were enforced on the type or age of participants included in each study.

Initial database searches identified 4225 articles. Application of exclusion criteria reduced the number of articles to 134 , which were further reduced to 25 by removing duplicates. An extensive review of the reference list of each included publication and hand searching identified 2 relevant articles. The search procedure identified 27 studies (Figure 1) that met criteria for inclusion in this review summarised in Table 1.

Healthy, able-bodied individuals can sit for lengthy periods without developing pressure-related injuries. Two studies measuring the frequency of seated movements found that able-bodied individuals, with no or little risk of developing pressure ulcers, make considerably more seated postural movements than the rate of seated postural movements recommended by the National Pressure Ulcer Advisory Panel [44].

Linder-Ganz et al. [23] identified that on average, 10 ablebodied participants, seated in a standard wheelchair, changed posture by approximately $15^{\circ}$ every $9 \pm 6$ minutes and $8^{\circ}$ every $6 \pm 2$ minutes in the sagittal and frontal planes, respectively. Unfortunately the value of seated postural movements for relieving or redistributing pressure at the seating interface was not recorded or documented by Linder-Ganz et al. [23]; however, a later study [32] reported that frequent postural movements when seated altered interface pressure and can restore blood flow, thus facilitating tissue health. This study found that 19 healthy able-bodied male subjects changed their posture on average $7.8 \pm 5.2$ times an hour in the frontal and sagittal planes when sitting in a wheelchair. Additionally, with each posture change, subcutaneous oxygen saturation increased on average by $2.2 \pm 2.4 \%$ [32].

Despite reporting that seated movements affected interface pressures, Reenalda et al. [32] did not document the actual changes in interface pressure. However, it can be deduced that a decrease in interface pressure occurred during posture shifts which allowed tissue oxygenation and perfusion to occur [3].

Although both studies report a favourable outcome for encouraging frequent postural movements to individuals at risk of developing pressure ulcers, their findings are limited to the results of able-bodied individuals, with healthy tissue; therefore, results are not generalisable to the wider population or those most at risk of developing pressure ulcers. Furthermore, it could be argued that the high rate of seated movements reported may have been to alleviate discomfort which is associated with high interface pressures [45] or unease of sitting in a chair of unusual choice for an ablebodied individual and fatigue from sitting over 60 minute [32] to 90 minute [23] period without any lower extremity movement [46]. However, both studies report similar rates of seated postural movements performed by healthy ablebodied individuals, which is in contrast to the rate of pressure

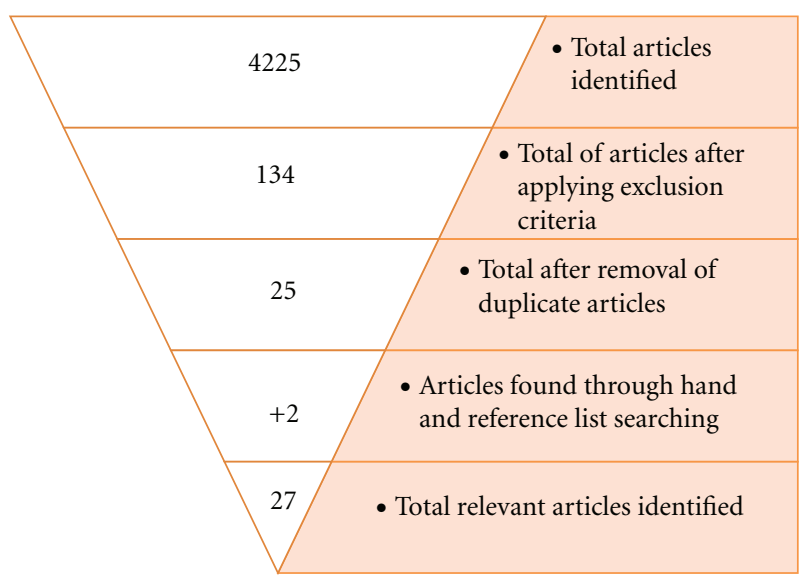

FIGURE 1: Flow diagram of search strategy.

relieving movements recommended to [44] and performed by those most at risk [43].

Yang et al. [43] demonstrated the infrequency with which 20 community dwelling manual wheelchair users with SCI engaged in pressure relieving movements, by remotely monitoring their daily sitting behaviours over a one-week period. Manual wheelchair users were found to spend an average of 9.2 hours (median 9.7, range 3.2-12.2 hours) per day in their own wheelchair and the average pressure relieving frequency was 9.4 times a day. Considering pressure relieving behaviour was defined as any lift off activity lasting longer than 10 seconds, [43] and as it has been reported that tissue reperfusion rates for this population can take up to 300 seconds [24], the quality of the movements in relation to pressure relief is questionable, thus illustrating the rarity of pressure relieving movements performed. Additionally, the average time of uninterrupted sitting was around 97 minutes (median 62, range 24-284), which indicates that participants in this study did not adhere to pressure relieving recommendations of relieving seated pressures as often as every 15 minutes [44].

It is of note that not all of the participants in this study used a pressure redistributing cushion despite the participants having a diagnosis of paraplegia $(n=11)$ or tetraplegia $(n=9)$ which would indicate that they were at increased risk of ulceration. Interestingly, participants with pressure redistributing cushions $(N=16)$ showed a significant increase in uninterrupted sitting time $(P=0.029)$ than those without pressure redistributing cushions $(N=$ 4) [46]. Considering that cushions, like human tissue, need a recovery period of off loading to return to their original form before they can be put under stress again highlights the increased level of risk that some of the participants were exposed to by not frequently engaging in pressure relieving movements.

Similarly, results from postal questionnaires investigating the preventative health behaviours and perceived risk of developing pressure ulcers of community dwelling wheelchair users [39] and persons with spinal cord injury 


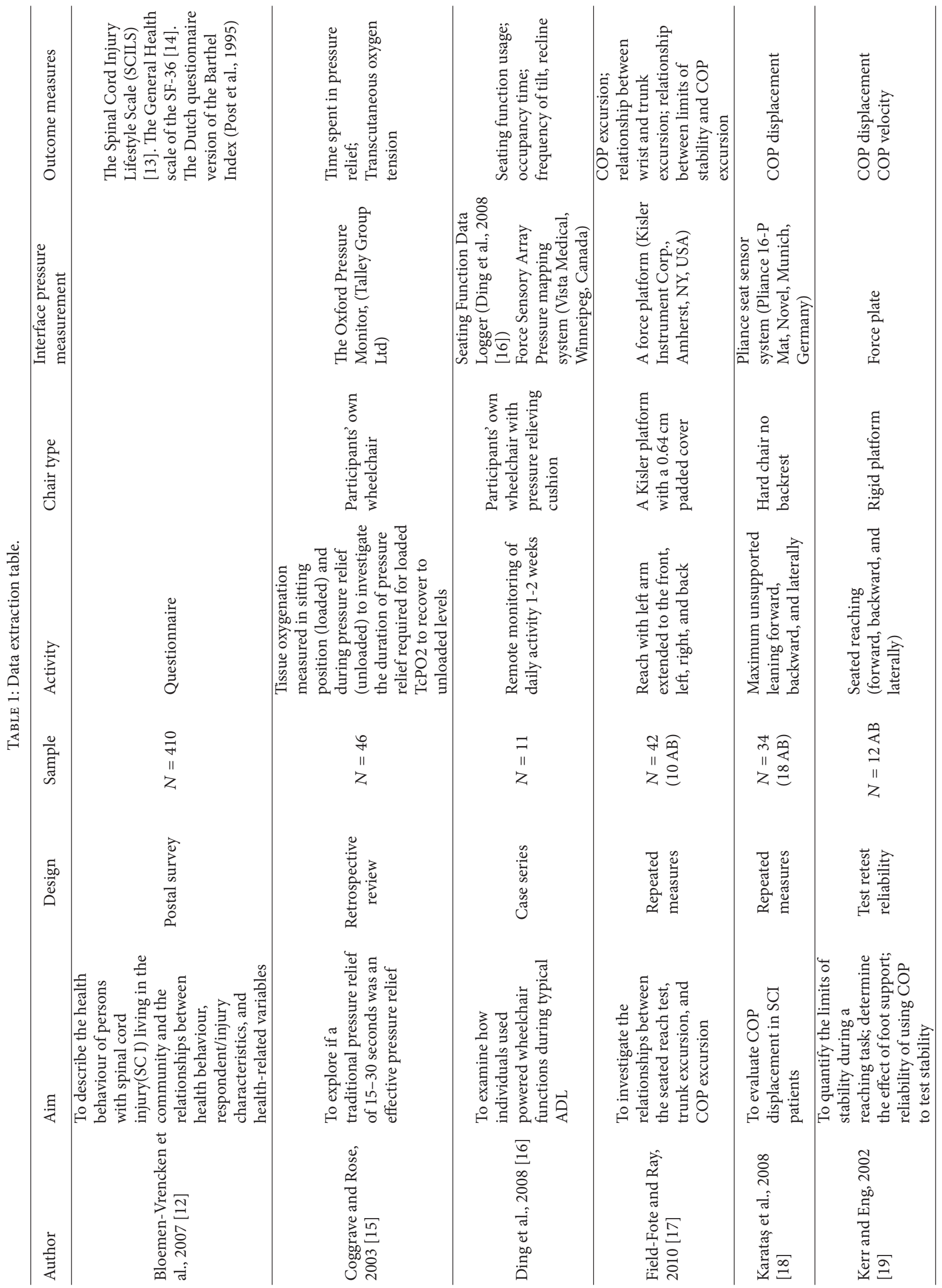




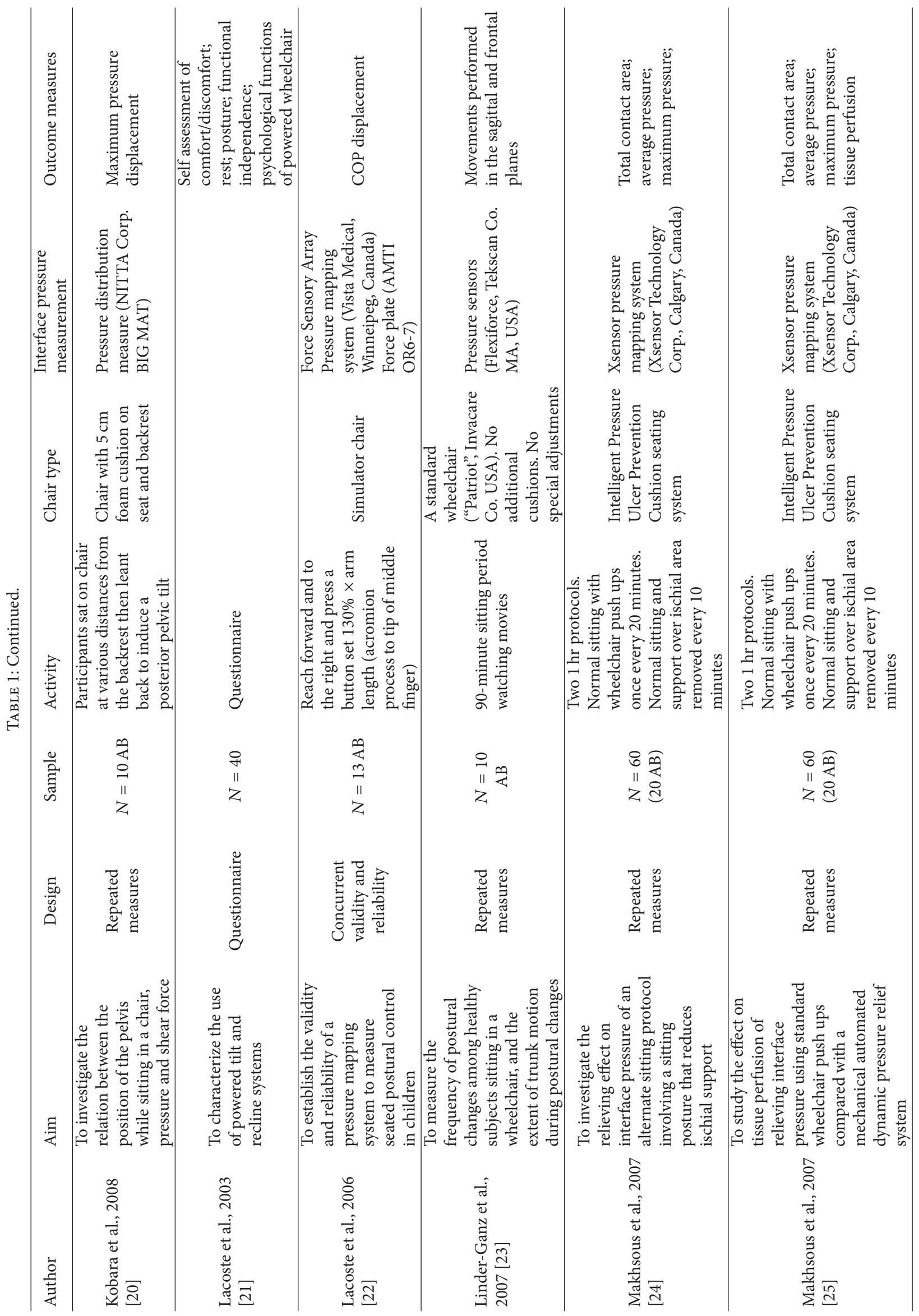




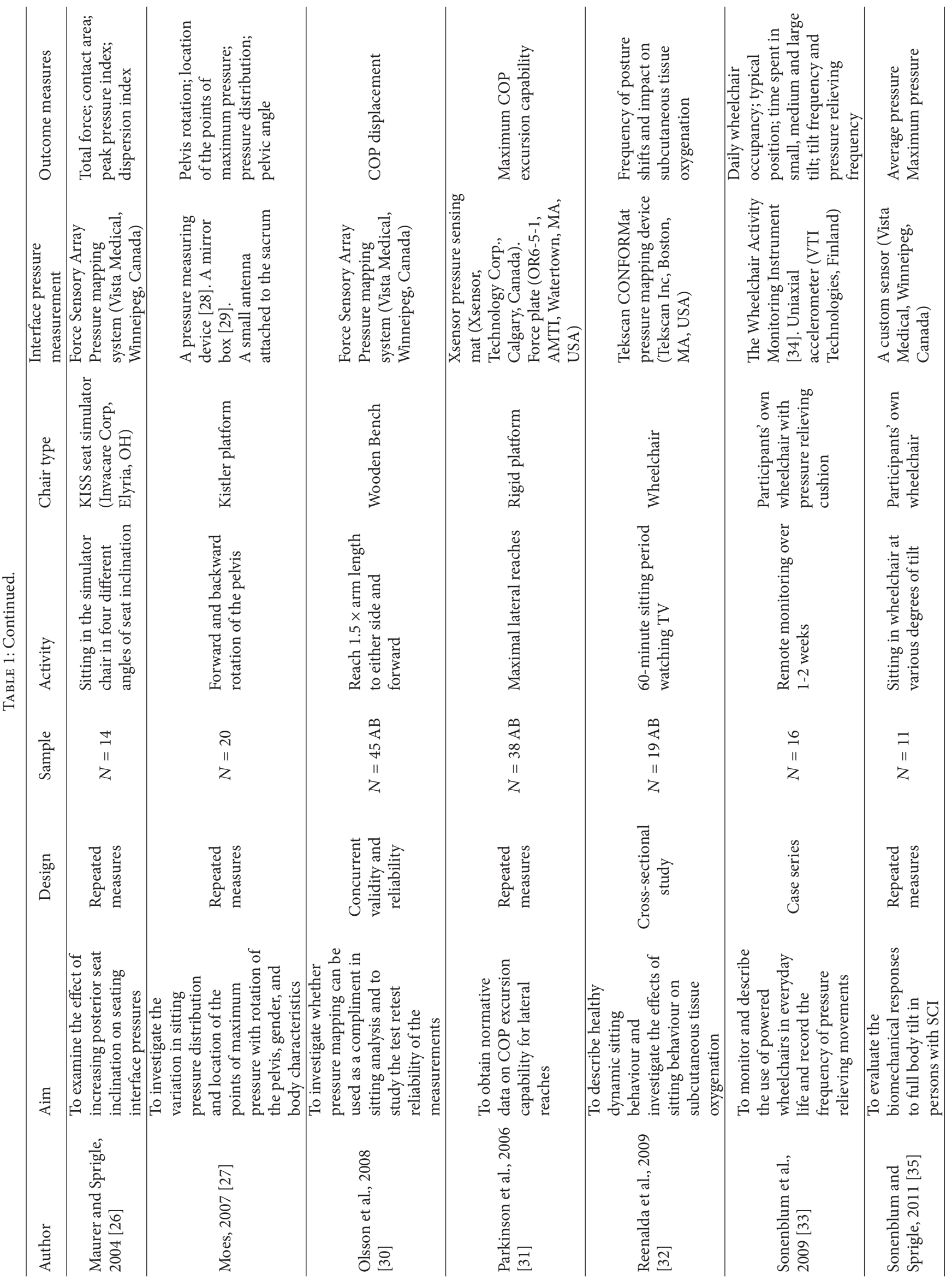




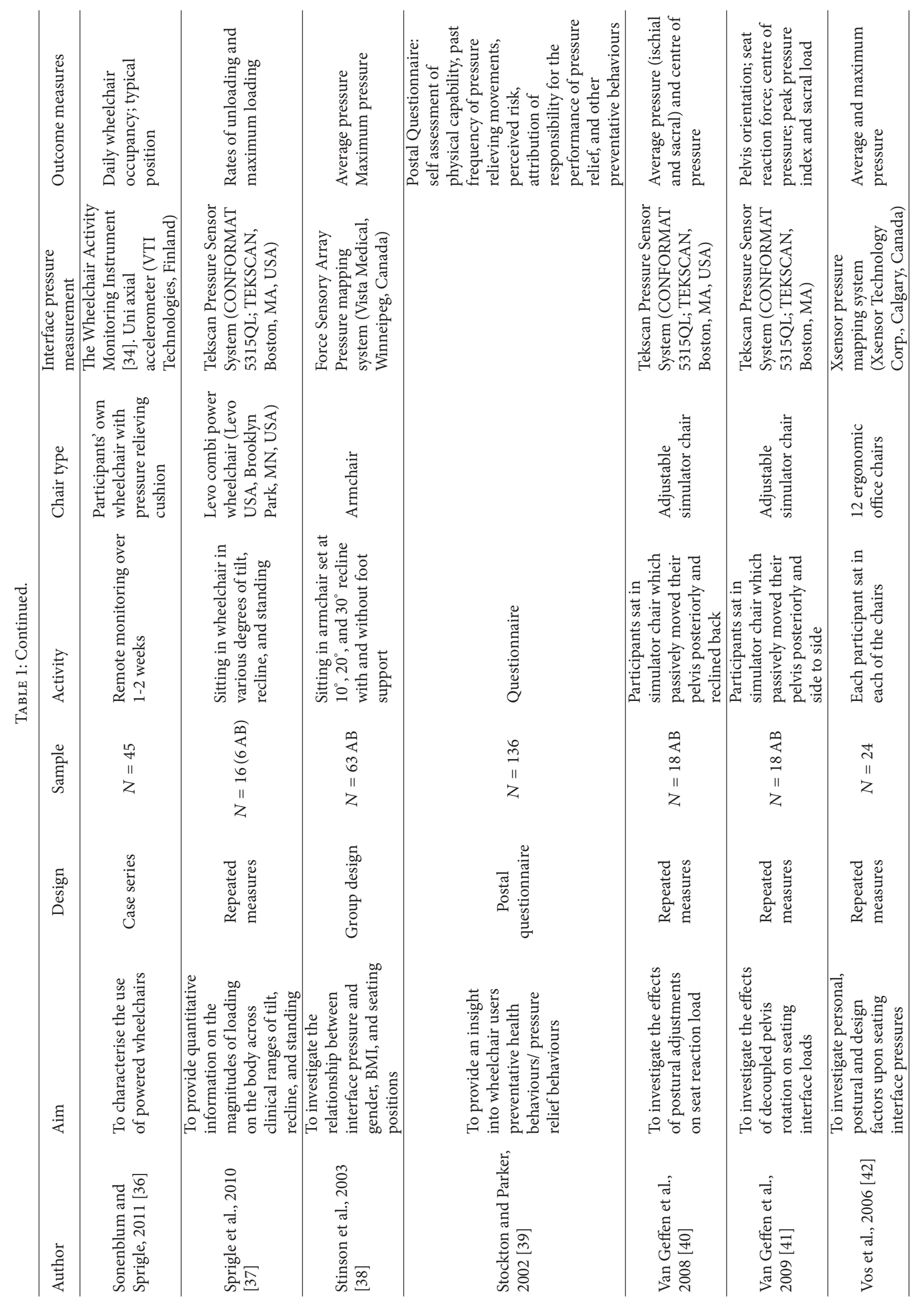




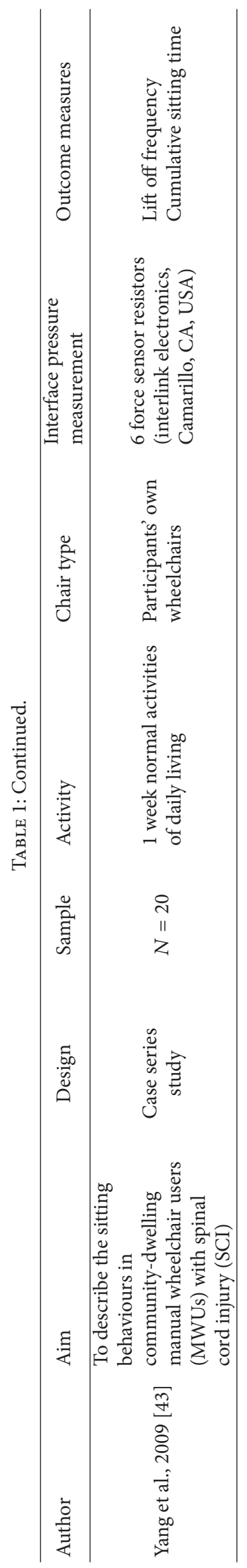


[12] identified a low performance rate of pressure relieving movements.

Bloemen-Vrencken et al. [12] found that over half of the 410 respondents did not engage in pressure relief movements frequently and only $20.9 \%$ of responders always did some type of pressure relief every 30 minutes. Likewise, Stockton and Parker [39] discovered that although $80 \%$ of their 136 wheelchair-dependent sample reported they were able to perform weight shifting movements, only $20.8 \%$ engaged in pressure relief activity once an hour and a further $54.7 \%$ moved less than once per hour, indicating a lack of adherence rather than ability.

Bloemen-Vrencken et al. [12] found that respondents who had experienced pressure ulcers implemented significantly more pressure ulcer prevention techniques such as relieving pressure regularly than those who had not experienced secondary complications (median total score of 256 respondents with no pressure sores $=45$, median total score of 154 respondents having experienced pressure sores in last 12 months $=48, P<0.05$ ). Conversely, Stockton and Parker [39] reported that respondents who had experienced a pressure ulcer did not significantly alter the frequency of pressure relieving movements performed.

Coggrave and Rose [15] identified ease of performance as the main reason few adhered to recommendations. This small-scale clinical study found that for 46 spinal cord injured participants, the mean duration of pressure relief required to raise tissue oxygen to unloaded levels was 1 minute 51 seconds (range 42 seconds- 3 minutes 30 seconds), which may be considered a long time to hold a pressure relieving position [15].

2.1. Key Message. Despite ability, it has been shown that very few manual wheelchair users adhere to the frequency of pressure reliefs recommended $[12,39,46]$. Policy guidance suggests that wheelchair users should be educated to perform pressure relieving movements regularly and be advised not to sit for longer than $2 \mathrm{hrs}$ in the same position [10].

\section{Tilt and Recline as Pressure Relief Movement}

Wheelchair tilt systems passively tilt the whole chair back, while maintaining a constant hip and knee angle. Depending on the extent of the tilt angle applied, wheelchair tilt systems can affect postural control, digestion, and reduce pressure around the vulnerable ischial tuberosity region by redistributing body weight from the seat to the backrest.

Two studies investigating the impact of tilt on blood flow and localised tissue loading for 11 individuals with SCI [35] and the load redistribution qualities of variable position wheelchairs with 6 able-bodied and 10 SCI individuals [37] found that the largest decrease of seating interface pressure occurred during larger tilts of up to $55^{\circ}$ from an upright sitting position $[35,37]$. As the ischial tuberosities are a curved structure, it may be postulated that a larger movement would be needed to offload the tissue around this area.

However, results are based on small study populations and equipment and seating conditions varied on both studies.
Sonenblum and Sprigle [35] used laser Doppler Flowmetry and a custom sensor from FSA (Vista Medical, Winnipeg, Canada) affixed to the skin at the apex of the ischial tuberosity region, whilst participants that sat in their own chair fitted with their prescribed pressure relieving cushion. Using a repeated measures design, each tilt was normalised against each starting position, and by not altering the participants original chair configuration, Sonenblum and Sprigle [35] attempted to create a more realistic interpretation of the effect of tilting on pressure and blood flow for wheelchair users in their "naturalistic" state.

By contrast, Sprigle et al. [37] utilized a single seating system to standardise all the support surfaces and seat and back articulations and placed pressure sensing mats (CONFORMAT 5315QL; TEKSCAN, Boston, MA, USA) below the cushions of the seat and backrest to monitor load redistribution during tilting. However, measuring average pressure over the entire mat may have confounded results, as during a tilt, gravitational forces may have influenced the pressure under the thigh area more than under the ischial area.

Despite methodological differences it is reassuring that both studies reported reasonably similar results indicating that tilting, as far as the seating system permits, reduced interface pressures.

Compared to tilting, reclining was found to unload the seating interface to a larger degree [37]. Recline increases the seat to back angle of a chair and can necessitate various positions from an upright seated position to a fully reclined supine position. Sprigle et al. [37] found that reclining the backrest fully to $90^{\circ}$ produced a $61 \%$ reduction on seat forces when compared to upright sitting for both able-bodied and SCI participants. This was to be expected, as lying back to $90^{\circ}$ effectively spreads the pressure over a larger surface area, compared to sitting; hence reclining was found to reduce interface pressures [37].

Similarly, Stinson et al. [38] found that greater recline angles reduced average seating interface pressures, when investigating the relationship of gender, body mass index, and seating position with seating interface pressures in ablebodied participants. Reclining the chair by $30^{\circ}$ was found to significantly reduce average pressure $(P<0.001)$. However, reclining $10^{\circ}, 20^{\circ}$, and $30^{\circ}$ did not significantly alter maximum pressure, which is the highest individual pressure recorded by a sensor over the entire pressure mat. Maximum pressure is considered an unstable measurement [47] as it focuses on the pressure recorded by one single sensor and therefore changes frequently, which may confound results.

Interestingly, armchair recline with feet supported was found to significantly increase average interface pressures [38]. However, results were based on measurements from able-bodied participants, known to have healthier, less atrophied tissue than individuals at risk of developing ulcers. Conversely, wheelchair recline with feet supported was found to stimulate greater unloading in a SCI cohort, who are physiologically predisposed to developing pressure ulcers than in a healthy able-bodied cohort $(P=0.003)$ [37].

As tilt and recline were both found to reduce pressure at the seating interface, it would be reasonable to assume 
that reducing the angle between the backrest and the seat surface to lower the rear portion of the seat surface (known as squeezing) would increase interface pressure. However, Maurer and Sprigle [26] found no evidence to suggest that "squeezing" a wheelchair frame to induce posterior seat inclination negatively affected seating interface pressures. As squeezing increased, more of the individuals came into contact with the seat surface; however, pressures around the ischial region remained the same thus limiting the value of "squeezing" the chair for relieving interface pressures.

3.1. Key Message. The studies found that increasing the angle of tilt and increasing recline reduce the loading of pressure at the seating interface. Policy guidelines suggest that education and training in the appropriate use of equipment to facilitate pressure relief should be given to both the individual at risk of developing the pressure ulcer and their caregivers [44].

\section{Pressure Relief Behaviour of Individuals with Tilt/Recline Systems}

Lacoste et al. [21] found that $97.5 \%$ of the 40 powered wheelchair users interviewed used their wheelchair daily, of which $\leq 35 \%$ used tilt or recline for physiological functions such as relieving pressure. Many of the respondents used the larger tilts $\left(31-45^{\circ}\right)$ for rest rather than pressure relief [21] questioning the practicalities of performing such a large movement regularly. Additionally, none of the respondents had a wheelchair that tilted beyond $45^{\circ}$, possibly explaining why among the few subjects who used their seating system for pressure relief, $\geq 50 \%$ used small amplitudes $\left(\leq 15^{\circ}\right)$ and $40-50 \%$ used medium amplitudes $\left(16-30^{\circ}\right)$ of tilt. However, Lacoste et al. [21] based their findings on the subjective opinion of 40 powered wheelchair users and recall bias and perception of illustrated seat angles may have clouded the accuracy of the findings reported.

Conversely, three studies, though methodologically different, also found that few subjects tilted past $45^{\circ}[16,33,36]$, despite all [33] or nearly all $[16,36]$ of the participants using a wheelchair that was capable of at least a $40^{\circ}$ tilt.

Ding et al. [16] reported that over a 2-week period, 11 subjects occupied their wheelchairs for $11.8 \pm 3.4$ hours, transferred in and out of their wheelchairs $5 \pm 5.3$ times, and performed $19 \pm 14$ tilts on average per day. Similarly, Sonenblum and Sprigle [36] and Sonenblum et al. [33] found that power wheelchair users occupied their wheelchairs an average of 11 hours [33] and 11.7 hours per day [36] and performed tilts $3.0 \pm 2.9$ times [36] and $4.3 \pm 3.9$ times per occupancy hour [33].

However, a change in tilt was classified as an angle change of $\geq 2.5^{\circ}$ that lasted for at least 1 minute [16] or any changes of more than $5^{\circ}$ held for more than 20 seconds $[33,36]$. The definition of a tilt or recline involving a minimal change in backrest angle may have overinflated results reported. However, Ding et al. [16] used an algorithm to filter raw data and reduce the likelihood of accidental fluctuations in backrest angle interfering with results.
Tilts and reclines of less than $20^{\circ}$ were found to be more frequent and held for a longer duration of time than larger tilts and reclines [16], though 2 of the 11 wheelchairs used in this study did not tilt past $20^{\circ}$, which may have skewed results. Similar findings were reported by Sonenblum and Sprigle [36] and Sonenblum et al. [33] as the subjects in these studies spent the majority of time in tilt angles $<20^{\circ}$, despite all [33] or nearly all [36] of the participants chairs having the capability of tilting to or past $45^{\circ}$. However, although participants were found to tilt relatively frequently, these tilts, according to the literature, were not large enough to relieve pressure around the vulnerable ischial tuberosity region $[37,38]$.

4.1. Key Message. The studies indicate that despite education, powered wheelchair users do not use the recommended magnitude of tilt or recline required to adequately relieve pressure around the vulnerable ischial tuberosity region. Interestingly, these larger magnitudes of tilt and recline were used to relieve pain and discomfort. Policy guidelines exemplify that although comfort is of primary importance, a flexible repositioning schedule based on the individual's preferences and tolerance should be encouraged as variable position seating is reported to redistribute pressures from around the vulnerable ischial region [44].

\section{Seat Adjustments to Induce Postural Movements}

Moes [27] explored the relationships between interface pressure, pelvic rotation, and body characteristics of 19 ablebodied participants. Through multiple regression techniques it was shown that intrinsic characteristics including ectomorphic index, gender, mass, and the body anthropometry greatly affected pressures at the seating interface during forward and backward rotations of the pelvis.

Similarly, Vos et al. [42] investigated postural and chair design effects on seating interface pressures using 12 ergonomic office chairs and 24 able-bodied participants (12 male; 12 female). They found that the greatest impacts on seating interface pressures were chair design differences, followed by participant characteristics such as gender, and lastly postural movements.

Both studies investigated a relatively small postural change in pelvic tilt [27] and pelvic and trunk tilt [42] which are known to have a minimal effect on interface pressures $[37,38]$. However, the slight pelvic movement was enough to effectively reduce the maximum pressure for a typically ectomorph (thin) person [27]. Similarly, Vos et al. [42] found that an increased posterior pelvic tilt significantly reduced pressure values for all participants.

Conversely, posterior pelvic tilt was found to have no significant effect on maximum pressure for 10 healthy male participants [20] and actually statistically increased the estimated shear force on the seat $(P<0.01)$. Shear force is the distortion of a body by two oppositely directed parallel forces; hence, such forces can damage skin and are known to contribute to pressure ulceration [44]. 
Kobara et al. [20] measured pressure distribution while each subject sat in the same chair, at various distances from the backrest and leant back, thus simulating a posterior pelvic tilt. The results showed that the locations of the points of maximum pressure were significantly displaced forward in all positions, upright $(P=0.005)$; sitting $5 \mathrm{cms}$ from the backrest $(P=0.008)$; sitting $10 \mathrm{~cm}$ from the backrest $(P=$ $0.285)$. This illustrated the element of shear in all positions and increasing posterior tilt which would increase the risk of pressure ulceration at these sites. Maximum pressure, as explained previously, is considered an unstable measure [47] and was used in this study to gauge the movement of the ischial tuberosities and not to investigate the redistribution of pressure during a pelvic movement. Additionally this study used a small able-bodied cohort and a chair with a hard surface with no alterations for subjects of varying morphologies, which may have confounded results.

Similar to Kobara et al. [20], Van Gefen et al. [40, 41] found that posterior pelvic tilt had a minimal effect on ischial pressures when investigating an experimental simulator chair that could independently control the orientation of the trunk, pelvis, and thighs with 18 healthy male subjects. It is possible that the exclusion of trunk movements limited the degree of posterior pelvic tilt, causing the ischial tuberosities to roll within their original area of high pressure and therefore minimally affecting overall pressure measurements.

Additionally, Van Geffen et al. [41] reported that a pelvic side elevation of $9^{\circ}$ reduced ischial pressure by $34 \%$, which was to be expected as the majority of body weight and therefore interface pressure were redistributed to the lower side of the chair surface. In other words, forcing a pelvic obliquity reduced pressure under the higher ischial tuberosity and undoubtedly increased pressure under the lower weight bearing side. This highlights the increased risk of ulceration individuals with a pelvic obliquity face and the importance of correct positioning and specialist seating prescription.

Makhsous et al. [24, 25] also evaluated the effect of chair design on interface pressures by periodically removing the ischial support area of a chair every 10 minutes for $10 \mathrm{~min}$ utes, compared to traditional pressure relieving movements (push up held for as long as possible or hoisting out of wheelchair for 60 seconds) every 20 minutes over two 1hour sitting periods using 60 participants (20 paraplegic; 20 tetraplegic; 20 able bodied).

Both studies reported that removal of the ischial support area of the chair significantly diminished interface pressures around this area and that a significant proportion of the buttock pressure was redistributed to the thigh area during these simulated pressure reliefs. Furthermore, Makhsous et al. [24] noted that of those who could perform push up pressure reliefs, the average push up time achieved was $49 \pm 2.8$ seconds; however, it took in the range of 200-300 seconds for tissue perfusion recovery to occur. Therefore, participants in this study were unable to hold the pressure relieving movements for long enough to allow tissue reperfusion to occur.

However, comparing a physical pressure relieving movement to a mechanical unloading of the ischial area for 10 minutes (600 seconds) may have skewed results in favour of the adjustable chair. Also, it is possible that removal of ischial support would cause an increase of pressure acting on the upper thigh area in contact with the remaining seat edge. Finally, the ease of removing the ischial section of the seat and the participant's views and dignity are not discussed; hence, further investigation is needed before definitive conclusions are made.

5.1. Key Message. These studies emphasise that larger postural movements are more effective in redistributing seating interface pressures. Policy guidelines recommend that clinicians should consider body size, body posture, mobility, lifestyle, and deformity when prescribing seating for individuals at risk of pressure ulceration [44]; hence, poor postural stability and mobility, for example, may indicate the need for a powered wheelchair device to access postures required for adequate pressure relief.

\section{Activity to Encourage Pressure Relieving Movements}

Postural stability is a prerequisite for the performance of seated movements and activities. Standing and seated stability are widely accepted to be measured by centre of pressure displacement $[17-19,22,30,31]$. The centre of pressure (COP) is the average location of the pressure, which has the potential to move as a person moves and adopts different positions.

Four studies considered the reliability and appropriateness of using pressure measurements in the form of COP for assessing seated static and dynamic stability with 12 healthy older adults [19]; 13 children aged $7-15$ years [22]; 45 children aged $4-15$ years [30]; 42 motor incomplete SCI adults and 10 healthy able-bodied adults [17].

Though studying different populations three of the studies employed a multidirectional reaching activity to investigate the repeatability of measuring COP for seating stability [17, 19, 30]. Kerr and Eng [19] measured COP displacement during multidirectional reaches with and without participants' feet being supported; Field-Fote and Ray [17] instructed participants to reach as far as possible with their right hand, while Olsson et al. [30] devised a fictional game where participants pretended to be an aeroplane with both arms out stretched to either side, then reached $1.5 \times$ the length of their arm (from 7 th cervical vertebra to the styloid process on the wrist) in each direction.

Lacoste et al. [22], on the other hand, instructed participants to reach 5 times to a target normalised to each participant's arm length (measured from acromion process to the tip of the middle finger $\times 130 \%$ ) in a forward direction and then to the side, to compare the static and dynamic measurements recorded by the Force Sensory Array pressure mapping system and a force platform (AMTI OR67). Fortunately, Lacoste et al. [22] found the pressure mapping system to be as effective as the force platform in detecting COP displacement; hence, results of all four studies can be discussed together $[17,19,22,30]$. 
Despite methodological and population differences, all four studies found the deviation of centre of pressure to be greater in the forward/backward movement than laterally $[17,19,22,30]$ and test re- test reliability was high for all directions of reach $[17,19,22]$. Greater COP displacement in the forward/backward direction was to be expected, as generally the base of support is larger in this direction (length of the thighs) compared to laterally (width of the hips).

Interestingly, Kerr and Eng [19] found that when reaching in the lateral and backwards direction, foot support significantly reduced COP displacements by $20 \%$; however, in contrast foot support increased COP displacement in the forward direction by $70 \%$. These phenomena may be explained by the supported feet extending the base of support in the forward direction, and limiting the counterbalancing effect of the feet when reaching laterally or backward.

Parkinson et al. [31] investigated the effect of having no foot support on the COP excursion capability of 38 ablebodied adults (age range from 21 to 74 years) and found that in forward, backward, and lateral reaching tasks, participants chose to swing their legs in the opposite direction of the reach. These results emphasise the counter balancing effect of the lower legs and feet during reaching tasks for able-bodied subjects and question the arc of reach accessible by those with limited lower limb function as the counter balancing effect of the lower limbs may be compromised.

Karatas et al. [18] found that the COP displacements for 16 SCI participants were significantly smaller in all directions (forward, backward, left, and right) than the measurements for 18 able-bodied subjects. The change in COP was expected to be smaller for the SCI cohort due to physiological differences in SCI individuals. However, it is possible that the ablebodied participants' ability to transfer weight through their lower limbs may have affected results.

Karatas et al. [18] found that SCI participants, with a history of pressure ulcers, had a smaller COP displacement than SCI participants with no history of pressure ulcers, which could not be explained by level of injury [18]. Therefore, it is possible that the ability to perform larger postural movements allows for more pressure relief and thus the group of SCI participants who could perform these movements had healthier tissue; however, as other interfacial pressures (such as peak pressure index, total contact area, and dispersion index) were not reported it is unwise to make a judgement.

6.1. Key Message. In sitting, COP indicates the degree of upper body movement available. These studies suggest that the greater the upper body movement, the greater the COP displacement and highlight the possibility that the pressure at the seating interface may also be affected. For ablebodied subjects, these studies found that the orientation of the feet in relation to the body impact on the degree of COP displacement available. Policy guidelines; however, for individuals at risk of pressure ulceration suggest that feet should be supported in sitting [44].

\section{Implications for Practice}

A review of the literature on the effectiveness of pressure relieving movements on seating interface pressures has shown that the majority of wheelchair users do not adhere with the recommended pressure relieving frequency or magnitude, even when they possess the ability to either physically or passively redistribute their body weight and hence reduce seating interface pressures and the likelihood of pressure ulcers occurring. Therefore, further research into the reasons behind the lack of concordance with pressure relieving recommendations amongst populations at risk should be explored. It is also recommended that more robust research into the effect of seated movements on interface pressures with populations at risk should be further investigated.

Until more detailed research is carried out, practitioners should continue to follow the current guidelines on pressure ulcer prevention such as the NICE "Pressure Ulcer Prevention and Treatment" guidelines [48] or the National and European Pressure Ulcer Advisory Panel guidelines [44] which both emphasise that practitioners should encourage clients to pressure relieve as regularly as possible.

\section{Conclusion}

None of the studies investigating functional activity and seating interface pressures explored the impact of these postural movements on the pressure around the vulnerable ischial region. Therefore, although it can be ascertained that functional activity influences the pressure at the seating interface, the positive or negative implications of such movements on seating interface pressures are currently not known.

This is particularly concerning as pressure ulcers are most likely to develop around the bony ischial tuberosity region and as the effect of activities on the pressure around this area is unknown, the performance of certain seated activities may aggravate the development of pressure ulcers for populations at most risk of developing such wounds. Hence further investigation into the effect of seated activities on interface pressures is necessary.

\section{Conflict of Interests}

The authors certify that no party having a direct interest in the results of the research supporting this paper has or will confer a benefit on them or on any organization with which the authors are associated; therefore, the authors certify that there is no conflict of interests known.

\section{Acknowledgment}

This work is supported by the Department for Employment and Learning.

\section{References}

[1] K. Vanderwee, M. H. F. Grypdonck, D. De Bacquer, and T. Defloor, "Effectiveness of turning with unequal time intervals 
on the incidence of pressure ulcer lesions," Journal of Advanced Nursing, vol. 57, no. 1, pp. 59-68, 2007.

[2] A. Gefen, "The biomechanics of sitting-acquired pressure ulcers in patients with spinal cord injury or lesions," International Wound Journal, vol. 4, no. 3, pp. 222-236, 2007.

[3] C. V. Bouten, C. W. Oomens, F. P. Baaijens, and D. L. Bader, "The etiology of pressure ulcers: skin deep or muscle bound?" Archives of Physical Medicine and Rehabilitation, vol. 84, no. 4, pp. 616-619, 2003.

[4] D. Bader, C. Oomens, F. Baaijens, and C. Bouten, "The aetiopathology of pressure ulcers: a hierarchical approach. Chapter 1," in Pressure Ulcer Research: Current and Future Perspectives, D. Bader, C. Bouten, D. Colin, and C. W. J. Oomens, Eds., Springer, Berlin, Germany, 2005.

[5] A. F. T. Mak, M. Zhang, and E. W. C. Tam, "Biomechanics of pressure ulcer in body tissues interacting with external forces during locomotion," Annual Review of Biomedical Engineering, vol. 12, pp. 29-53, 2010.

[6] J. L. Prentice and M. C. Stacey, "Pressure ulcers: the case for improving prevention and management in Australian health care settings," Primary Intention, vol. 9, no. 3, pp. 111-120, 2001.

[7] C. VanGilder, G. MacFarlane, S. Meyer, and C. Lachenbruch, "Body mass index, weight, and pressure ulcer prevalence: an analysis of the 2006-2007 international pressure ulcer prevalence ${ }^{\mathrm{TM}}$ surveys," Journal of Nursing Care Quality, vol. 24, no. 2, pp. 127-135, 2009.

[8] S. Sprigle and S. Sonenblum, "Assessing evidence supporting redistribution of pressure for pressure ulcer prevention: a review," Journal of Rehabilitation Research and Development, vol. 48, no. 3, pp. 203-213, 2011.

[9] A. Rose and L. MacKenzie, “'Beyond the cushion': a study of occupational therapists' perceptions of their role and clinical decisions in pressure care," Disability and Rehabilitation, vol. 32, no. 13, pp. 1099-1108, 2010.

[10] National Pressure Ulcer Advisory Panel, Treatment of Pressure Ulcers: Quick Reference Guide, National Pressure Ulcer Advisory Panel, Washington, DC, USA, 2009.

[11] European Pressure Ulcer Advisory Panel, Prevention and Treatment of Pressure Ulcers: Quick Reference Guide, National Pressure Ulcer Advisory Panel, Washington, DC, USA, 2009.

[12] J. H. A. Bloemen-Vrencken, L. P. De Witte, M. W. M. Post, and W. J. A. Van Den Heuvel, "Health behaviour of persons with spinal cord injury," Spinal Cord, vol. 45, no. 3, pp. 243-249, 2007.

[13] S. D. Pruitt, D. R. Wahlgren, J. E. Epping-Jordan, and A. L. Rossi, "Health behaviour in persons with spial cord injury: development and initial validity of an outcome measure," Spinal Cord, vol. 36, no. 10, pp. 724-731, 1998.

[14] J. Ware Jr and C. D. Sherbourne, "The MOS 36-item shortform health survey (SF-36): I. Conceptual framework and item selection," Medical Care, vol. 30, no. 6, pp. 473-483, 1992.

[15] M. J. Coggrave and L. S. Rose, "A specialist seating assessment clinic: changing pressure relief practice," Spinal Cord, vol. 41, no. 12, pp. 692-695, 2003.

[16] D. Ding, E. Leister, R. A. Cooper et al., "Usage of tilt-in-space, recline, and elevation seating functions in natural environment of wheelchair users," Journal of Rehabilitation Research and Development, vol. 45, no. 7, pp. 973-984, 2008.

[17] E. C. Field-Fote and S. S. Ray, "Seated reach distance and trunk excursion accurately reflect dynamic postural control in individuals with motor-incomplete spinal cord injury," Spinal Cord, vol. 48, no. 10, pp. 745-749, 2010.
[18] G. K. Karataş, A. K. Tosun, and U. Kanatli, "Center-of-pressure displacement during postural changes in relation to pressure ulcers in spinal cord-injured patients," American Journal of Physical Medicine and Rehabilitation, vol. 87, no. 3, pp. 177-182, 2008.

[19] H. M. Kerr and J. J. Eng, "Multidirectional measures of seated postural stability," Clinical Biomechanics, vol. 17, no. 7, pp. 555-557, 2002.

[20] K. Kobara, A. Eguchi, S. Watanabe, and K. Shinkoda, "The influence of the distance between the backrest of a chair and the position of the pelvis on the maximum pressure on the ischium and estimated shear force., Disability and Rehabilitation, vol. 3, no. 5, pp. 285-291, 2008.

[21] M. Lacoste, R. Weiss-Lambrou, M. Allard, and J. Dansereau, "Powered tilt/recline systems: why and how are they used?" Assistive Technology, vol. 15, no. 1, pp. 58-68, 2003.

[22] M. Lacoste, M. Therrien, J. N. Côté, I. Shrier, H. Labelle, and F. Prince, "Assessment of seated postural control in children: comparison of a force platform versus a pressure mapping system," Archives of Physical Medicine and Rehabilitation, vol. 87, no. 12, pp. 1623-1629, 2006.

[23] E. Linder-Ganz, M. Scheinowitz, Z. Yizhar, S. S. Margulies, and A. Gefen, "How do normals move during prolonged wheelchair-sitting?" Technology and Health Care, vol. 15, no. 3, pp. 195-202, 2007.

[24] M. Makhsous, M. Priebe, J. Bankard et al., "Measuring tissue perfusion during pressure relief maneuvers: insights into preventing pressure ulcers," Journal of Spinal Cord Medicine, vol. 30, no. 5, pp. 497-507, 2007.

[25] M. Makhsous, D. M. Rowles, W. Z. Rymer et al., "Periodically relieving ischial sitting load to decrease the risk of pressure ulcers," Archives of Physical Medicine and Rehabilitation, vol. 88, no. 7, pp. 862-870, 2007.

[26] C. L. Maurer and S. Sprigle, "Effect of seat inclination on seated pressures of individuals with spinal cord injury," Physical Therapy, vol. 84, no. 3, pp. 255-261, 2004.

[27] N. C. C. M. Moes, "Variation in sitting pressure distribution and location of the points of maximum pressure with rotation of the pelvis, gender and body characteristics," Ergonomics, vol. 50, no. 4, pp. 536-561, 2007.

[28] C. C. M. Moes, "Calibration of a pressure distribution measuring device," Technical Report, Delft University of Technology, Delft, the Netherlands, 1999.

[29] J. D. Chodera and M. Lord, "Paedobarographic foot pressure measurements and their applications," in Proceedings of the Strathclyde Bioengineering Seminar on Disability, P. H. Kenedi, Ed., pp. 173-181, University of Strathclyde, Glasgow, Scotland, McMillan Press, London, UK, August 1978.

[30] K. Olsson, A. Blomkvist, and E. Beckung, "Pressure mapping as a complement in clinical sitting analysis in children during activity," Advances in Physiotherapy, vol. 10, no. 2, pp. 76-84, 2008.

[31] M. B. Parkinson, D. B. Chaffin, and M. P. Reed, "Center of pressure excursion capability in performance of seated lateralreaching tasks," Clinical Biomechanics, vol. 21, no. 1, pp. 26-32, 2006.

[32] J. Reenalda, P. Van Geffen, M. Nederhand, M. Jannink, M. Ijzerman, and H. Rietman, "Analysis of healthy sitting behavior: interface pressure distribution and subcutaneous tissue oxygenation," Journal of Rehabilitation Research and Development, vol. 46, no. 5, pp. 577-586, 2009. 
[33] S. E. Sonenblum, S. Sprigle, and C. L. Maurer, "Use of power tilt systems in everyday life," Disability and Rehabilitation: Assistive Technology, vol. 4, no. 1, pp. 24-30, 2009.

[34] S. E. Sonenblum, S. Sprigle, F. H. Harris, and C. L. Maurer, "Characterization of power wheelchair use in the home and community," Archives of Physical Medicine and Rehabilitation, vol. 89, no. 3, pp. 486-491, 2008.

[35] S. E. Sonenblum and S. H. Sprigle, "The impact of tilting on blood flow and localized tissue loading," Journal of Tissue Viability, vol. 20, no. 1, pp. 3-13, 2011.

[36] S. E. Sonenblum and S. Sprigle, "Distinct tilting behaviours with power tilt-in-space systems," Disability and Rehabilitation: Assistive Technology, vol. 6, no. 6, pp. 526-535, 2011.

[37] S. Sprigle, C. Maurer, and S. E. Sorenblum, "Load redistribution in variable position wheelchairs in people with spinal cord injury," Journal of Spinal Cord Medicine, vol. 33, no. 1, pp. 58-64, 2010.

[38] M. D. Stinson, A. Porter-Armstrong, and P. Eakin, "Seatinterface pressure: a pilot study of the relationship to gender, body mass index, and seating position," Archives of Physical Medicine and Rehabilitation, vol. 84, no. 3, pp. 405-409, 2003.

[39] L. Stockton and D. Parker, "Pressure relief behaviour and the prevention of pressure ulcers in wheelchair users in the community.", Journal of Tissue Viability, vol. 12, no. 3, pp. 84-92, 2002.

[40] P. van Geffen, J. Reenalda, P. H. Veltink, and B. F. J. M. Koopman, "Effects of sagittal postural adjustments on seat reaction load," Journal of Biomechanics, vol. 41, no. 10, pp. 2237-2245, 2008.

[41] P. van Geffen, J. Reenalda, P. H. Veltink, and B. F. J. M. Koopman, "Decoupled pelvis rotation in sitting: a passive motion technique that regulates buttock load associated with pressure ulcer development," Journal of Biomechanics, vol. 42, no. 9, pp. 1288-1294, 2009.

[42] G. A. Vos, J. J. Congleton, J. Steven Moore, A. A. Amendola, and L. Ringer, "Postural versus chair design impacts upon interface pressure," Applied Ergonomics, vol. 37, no. 5, pp. 619-628, 2006.

[43] Y. S. Yang, G. L. Chang, M. J. Hsu, and J. J. Chang, "Remote monitoring of sitting behaviors for community-dwelling manual wheelchair users with spinal cord injury," Spinal Cord, vol. 47, no. 1, pp. 67-71, 2009.

[44] National Pressure Ulcer Advisory Panel, Pressure Ulcer Prevention Points, National Pressure Ulcer Advisory Panel, Washington, DC, USA, 2007.

[45] G. Kyung and M. A. Nussbaum, "Driver sitting comfort and discomfort (part II): relationships with and prediction from interface pressure," International Journal of Industrial Ergonomics, vol. 38, no. 5-6, pp. 526-538, 2008.

[46] K. Uenishi, M. Tanaka, H. Yoshida, S. Tsutsumi, and N. Miyamoto, "Driver's fatigue evaluation during long term driving for automotive seat development," SAE Technical Paper Series 2002-01-0773, 2002.

[47] S. H. Sprigle, T. E. Faisant, and K. C. Chung, "Clinical evaluation of custom-contoured cushions for the spinal cord injured," Archives of Physical Medicine and Rehabilitation, vol. 71, no. 9, pp. 655-658, 1990.

[48] NICE Clinical Guideline 29, Pressure Ulcer Prevention, National Institute of Clinical Excellence, London, UK, 2005. 


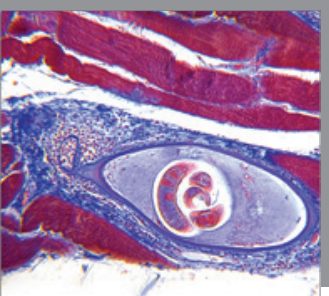

Gastroenterology

Research and Practice
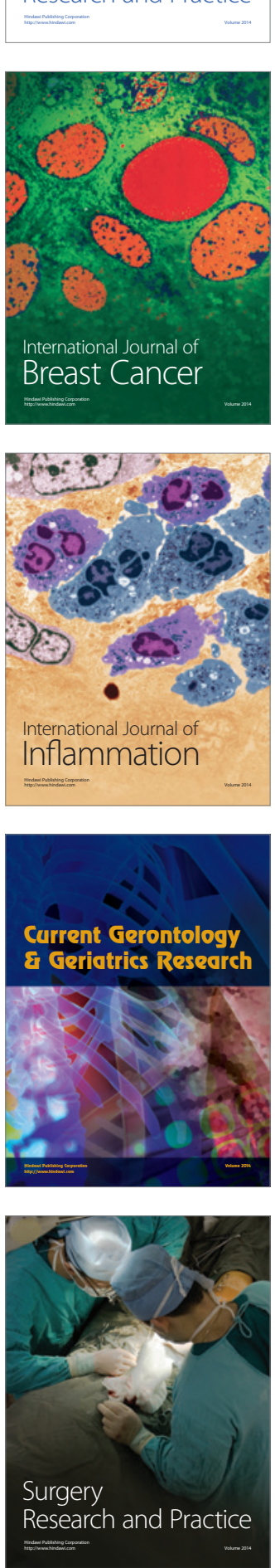

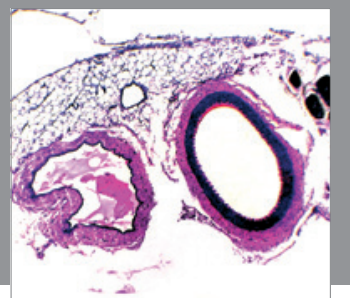

International Journal of Hypertension
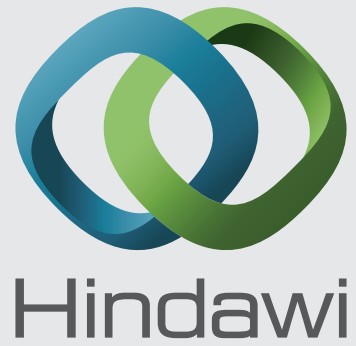

Submit your manuscripts at http://www.hindawi.com
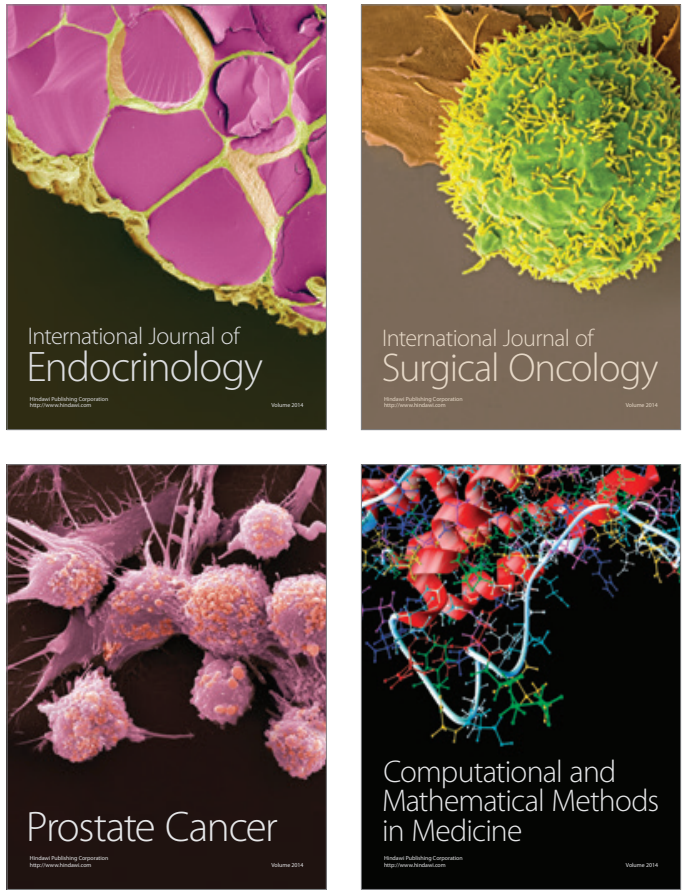
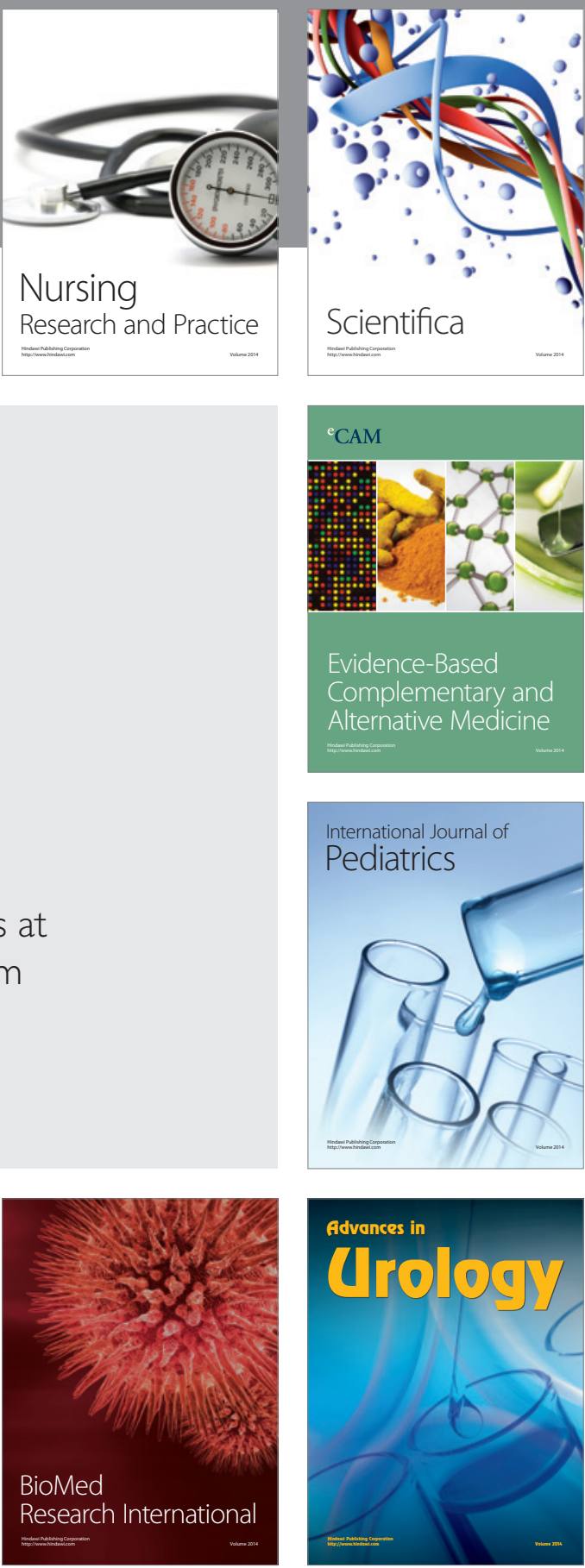

Nursing

Research and Practice

Scientifica

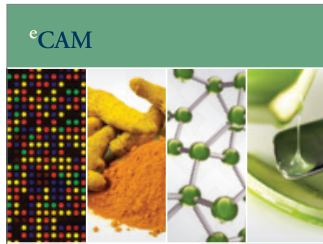

Evidence-Based

Complementary and Alternative Medicine
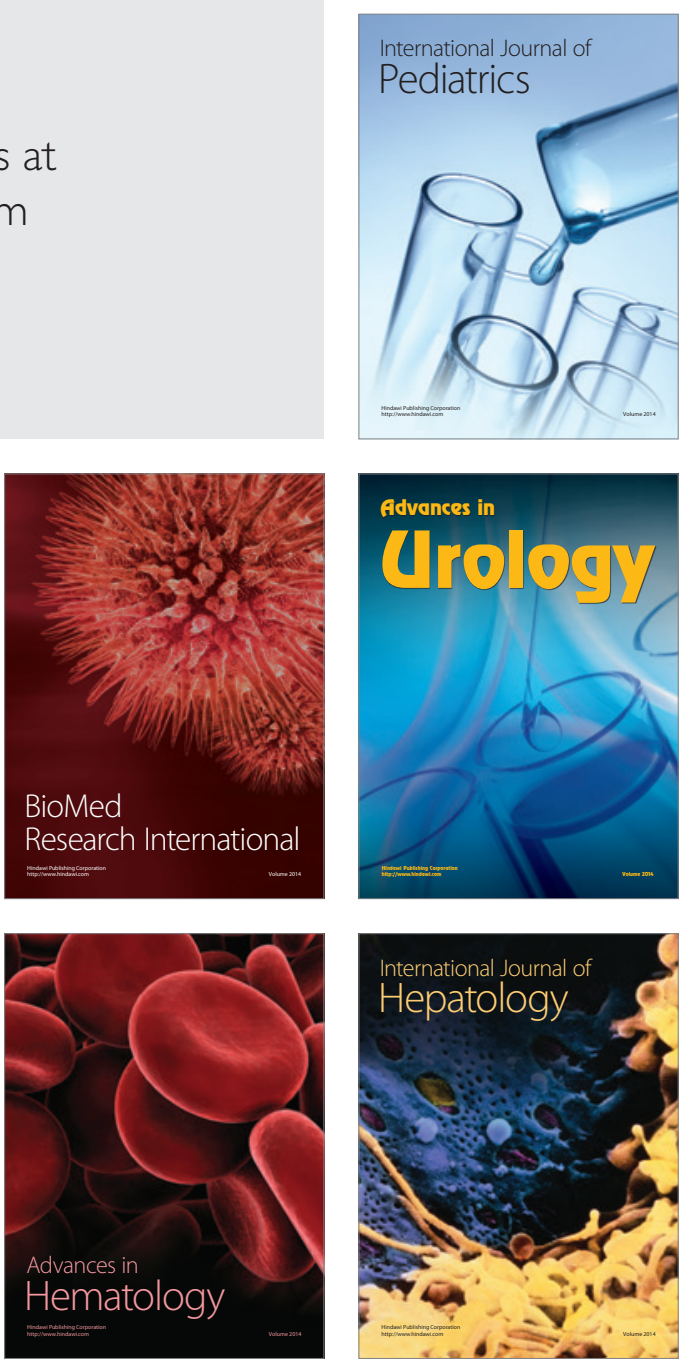